\title{
Nature Neuroscience offers open access publishing
}

The authors of primary research papers accepted by our journal have the opportunity to make their work freely available to all upon publication.

U ntil last year, Nature Neuroscience operated solely under a traditional subscription model, wherein the cost of publication was covered by charging for access to our content. Beginning in January 2021, we and our sister journals, including Nature, became 'transformative' journals. This means that authors of accepted primary research articles can now choose to have their papers published under a 'gold' open access model, in which the final version of a paper is made freely available (under a CC BY license) immediately upon publication. In this scenario, the cost of publication is covered by an Article Processing Charge (APC) paid at the time of publication. The APC for Nature Neuroscience in 2022 is €9,500/US $\$ 11,390 / £ 8,290$, and more information can be found here.

This change is intended to be a complete transformation; the Nature Portfolio journals are committed to continuously increasing open access uptake, with the aim of transitioning to a fully open access model for primary research articles when there is sufficient demand. Commissioned and opinion content will remain accessible by subscription only.

This transition toward open access publishing reflects our broader commitment to open science and our desire for the content we publish to be widely accessible. It also aligns with strong demand from the scientific community, including individual researchers, funding agencies, professional societies, and institutional libraries. Of particular note, our transformative model is compliant with Plan S, the mandate for open access publishing laid out by cOAlition S, a large group of governmental and philanthropic funders. Thus, authors whose research is supported by these sources need not compromise on publishing their papers in Nature Portfolio journals to satisfy the need for open access. In addition, a 'transformative agreement' has been reached with the Max Planck Digital Library that establishes a single annual fee to cover APCs for affiliated authors as well as journal subscriptions. This type of agreement removes the burden of paying APCs from individual investigators, and we anticipate that more institutions will opt for this route in the coming years.

Open access publishing is advantageous to authors and to society as a whole. A 2018 study conducted by our parent company, Springer Nature, found that, of papers published in hybrid journals (those with both open access and paywalled options), those published open access received more downloads and citations and attracted more attention from news outlets and policy makers. More importantly, open access publishing is the right thing to do. The majority of scientific research is publicly funded, and as such its end products should be publicly available. Importantly, papers that are free to access may also be more likely to be taught in classes, particularly at high school and undergraduate levels where journal subscriptions may be beyond reach.

We, the editorial team of Nature Neuroscience, are enthusiastic about this transition and agree that open access to primary content is and should be the future of scientific publishing. That said, we want to be clear that editors receive no financial or professional benefit when authors opt for open access publishing, and that it plays no role in our editorial decision-making. In fact, the authors' decision about whether to publish open access is usually finalized only after a paper is accepted.

We understand that for many investigators, it will not yet be feasible to pay the APC from their grant funds. As mentioned earlier, some APCs are covered via transformative agreements, and we hope that the proliferation of these agreements will bring open access publishing within reach for more authors. The subscription option will remain available to our authors who do not have access to funding to cover APCs. Meanwhile, our fully open access sister titles, such as Nature Communications, offer a funding support service for authors who are unable to cover the cost of publication. Our aim is to ensure that lack of funds is never a barrier to publishing with us.

The landscape of science communication is changing rapidly, and Nature Neuroscience is changing with it. As editors, we feel strongly that our work - curating our content, working with authors to develop papers, and providing an appealing and highly visible final product - benefits the neuroscience community. We look forward to making articles in our journal available to many more readers in the years to come.

Published online: 6 January 2022 https://doi.org/10.1038/s41593-021-00995-2 